\title{
Is COVID-19 a risk factor for progression of benign prostatic hyperplasia and exacerbation of its related symptoms?: a systematic review
}

\author{
Abdolreza Haghpanah $\mathbb{1}^{1,2} \cdot$ Fatemeh Masjedi $\mathbb{D}^{1} \cdot$ Mehdi Salehipour $\mathbb{D}^{2} \cdot$ Alireza Hosseinpour $\mathbb{D}^{1,3} \cdot$ \\ Jamshid Roozbeh (10) ${ }^{1}$ Anahita Dehghani (i) ${ }^{1}$
}

Received: 17 November 2020 / Revised: 28 April 2021 / Accepted: 30 April 2021 / Published online: 18 May 2021

(c) The Author(s), under exclusive licence to Springer Nature Limited 2021

\begin{abstract}
Background To explore the potential mechanisms of SARS-CoV-2 in targeting the prostate gland, leading to exacerbation of benign prostatic hyperplasia (BPH) symptoms and greater risks of BPH complications such as acute urinary retention.

Methods A categorized and comprehensive search in the literature has been conducted by 10 April 2021 using international databases including PubMed, Embase, Web of Science, Scopus, and Cochrane Library in line with the PRISMA guidelines recommendations. PICO strategy was used to formulate the research question. The following terms were used: urology, COVID-19, coronavirus, BPH, inflammation, androgen receptors, LUTS, IPSS, PSA, and SARS-CoV-2 or a combination of them. Studies with irrelevant purposes and duplicates were excluded. The selected studies were performed on humans and published in English.

Results The research revealed 89 articles. After title screening and considering exclusion criteria, 52 papers were included for the systematic review. BPH is a common condition affecting older men. SARS-CoV-2 infects the host cell by binding to angiotensin converting enzyme 2 (ACE2). A hyperactivated RAS system during infection with SARS-CoV-2 may lead to activation of pro-inflammatory pathways and increased cytokine release. Thus, this virus can lead to exacerbation of lower urinary tract symptoms (LUTS) and trigger inflammatory processes in the prostate gland. Since androgen receptors (AR) play an important role in the BPH pathophysiology and infection with SARS-CoV-2 may be androgen-mediated, BPH progression and its related symptoms can be a complication of COVID-19 through AR involvement and metabolic disturbances.

Conclusions Based on the current findings, SARS-CoV-2 can possibly damage the prostate and worsen BPH and its related LUTS through ACE2 signaling, AR-related mechanisms, inflammation, and metabolic derangement. We encourage future studies to investigate the possible role of COVID-19 in the progression of BPH-related LUTS and examine the prostatic status in susceptible patients with relevant available questionnaires (e.g., IPSS) and serum biomarkers (e.g., PSA).
\end{abstract}

Fatemeh Masjedi

masjedi_f@sums.ac.ir

1 Shiraz Nephro-Urology Research Center, Shiraz University of Medical Sciences, Shiraz, Iran

2 Department of Urology, School of Medicine, Shiraz University of Medical Sciences, Shiraz, Iran

3 Student Research Committee, Shiraz University of Medical Sciences, Shiraz, Iran

\section{Introduction}

The outbreak of the ongoing global Coronavirus disease 2019 (COVID-19) pandemic that was caused by Severe acute respiratory syndrome coronavirus 2 (SARS-CoV-2) was first identified in late 2019 in Wuhan, China; since then, the world has faced a great global catastrophe; this life-threatening crisis has proven challenging to overcome [1]. The most common manifestation of the novel coronavirus seems to be pneumonia [2]; however, it is now widely accepted that the virus can present with symptoms outside the respiratory tract including digestive tract symptoms such as nausea, vomiting, and diarrhea [3]. Angiotensin-converting enzyme 2 (ACE2) appears to be a 
receptor for SARS-CoV-2, to which the virus binds to, enters, and infects the host cell [4]. Moreover, recent studies have found that co-expression of ACE2 and TMPRSS2 in an organ is crucial for the virus to be able to infect the organ [5]. Previously, it was believed that the virus mainly infected the lungs although discovering the co-expression of ACE2 and TMPRSS2 in other organs such as the kidneys, testes, and prostate raises the question of whether or not the virus can affect the aforementioned organs [6].

Benign prostatic hyperplasia (BPH) is a histologic diagnosis defined as excessive growth of the epithelial and stromal cells located in the transition zone of the prostate gland. BPH is the most common cause of benign prostatic enlargement (BPE), benign prostatic obstruction, and bladder outlet obstruction in older men [7-9]. Lower urinary tract symptoms (LUTS) are considered a consequence of a wide range of etiologies such as BPH and non-prostatic conditions including dysfunction of the bladder [10, 11]. Furthermore, international prostate symptom score (IPSS), as a validated questionnaire, and serum prostate-specific antigen (PSA) measurement are considered to evaluate the patients with $\mathrm{BPH}[10,12]$.

There are different risk factors related to the development of BPH such as LUTS, prostate growth, old age, sex-related hormones, and chronic inflammation [13]. The prevalence of $\mathrm{BPH}$ is increased in an age-dependent manner. It is estimated that $8 \%$ and $50 \%$ of male population at the 4 th and 6 th decade of life, respectively, are diagnosed with pathological BPH [14].

Recent studies have shown that males are more susceptible to SARS-CoV-2 infection and elder population seems to develop more severe cases of COVID-19 and subsequently they are more susceptible to hospitalization $[15,16]$. Furthermore, a significant number of patients with COVID-19 are asymptomatic carriers [17, 18]. As mentioned before, BPH increases in an age-dependent manner and then, a considerable number of older males are diagnosed with BPE [14]. Thus, one can hypothesize that a remarkable group of older male patients with COVID-19 including severe cases may have BPH as a comorbid condition and this condition may be exacerbated by COVID19. Recently, emerging studies have proposed that LUTS may be increased early symptoms of COVID-19 and IPSS, especially in older males, may be possible complications of this disease [19, 20]. To date, no study has investigated the potential mechanisms of SARS-CoV-2 in causing BPHrelated complications, LUTS or exacerbation of a previously diagnosed BPE in severe cases, and asymptomatic carriers of COVID-19 and the underlying mechanisms resulting in this condition as a complication of COVID-19 are not elucidated.

In this review, the main purpose was to explore the potential mechanisms of SARS-CoV-2 in targeting the prostate gland, leading to progression of $\mathrm{BPH}$, or exacerbation of its related LUTS.

\section{Materials and methods}

\section{Search strategy and selection criteria}

A categorized and comprehensive search in the literature was conducted by 10 April 2021 using international databases including PubMed, Embase, Web of Science, Scopus, and Cochrane Library in line with the recommendations from the Preferred Reporting Items for Systematic Reviews and Meta Analyses (PRISMA) guidelines [21]. All the records in English were included for investigating their eligibility, both published and peer-reviewed on-line publications. Additional sources were identified from citations of the retrieved literature. There was no limitation on sample size. Published reports of experiences with the SARS-CoV-2 have increased greatly in the past months, but no randomized trials to date regarding possible treatments have been identified regarding possible treatments. Most reports related to $\mathrm{BPH}$ or male prostate problems involved small cohorts, case reports, and editorials. Similarly, prior publications of other coronaviruses were limited in number. We reviewed all the titles for considering their eligibility. Full-text articles were then reviewed and evaluated for inclusion by authorship teams. The authors acknowledge that the number of publications about the novel SARSCoV-2 is increasing at an exponential rate and there may be bias toward reporting of positive findings. We used a broad inclusive search strategy in order to prevent missing any relevant records. Two experienced investigators conducted the systematic search. Population, intervention, comparison, and outcome strategy was used to formulate the research question: "Are BPH patients infected with SARS-CoV-2 at greater risk of developing LUTS and BPH-related complications compared to the normal population?" (Table 1). The following phrases were searched in different databases: "severe acute respiratory syndrome coronavirus 2," "2019 nCoV," "SARS-CoV-2," "coronavirus," "COVID-19," "benign prostatic hyperplasia," "inflammation," "androgen

Table 1 PICO strategy was used to formulate the research question: "Are BPH patients infected with SARS-CoV-2 at greater risk of developing LUTS and BPH-related complications compared to the normal population?".

\begin{tabular}{ll}
\hline P (Population) & Patients with BPH \\
$\begin{array}{l}\text { I (Intervention or } \\
\text { exposure) }\end{array}$ & Infection with SARS-CoV-2 \\
C (Comparison) & Normal population \\
O (Outcome) & $\begin{array}{l}\text { Lower urinary tract symptoms (LUTS) } \\
\text { and BPH-related complications }\end{array}$ \\
\hline
\end{tabular}


receptors," "lower urinary tract symptoms," "bladder outlet obstruction," "benign prostatic enlargement," "international prostate symptom score," "prostate specific antigen," "cytokine storm," "angiotensin-converting enzyme 2 receptor," "ACE2," "5- $\alpha$ reductase inhibitor," and "prostate involvement" or a combination of them in the titles/ abstracts.

\section{Data extraction}

Two authors (AH and FM) independently screened for inclusion, using the pre-specified criteria. If it was clear from the abstract that the study did not meet the selection criteria through reviewing the abstract, it was excluded. If it was unclear, the full paper was retrieved. Then, for relevant records, the full text was evaluated; discrepancies were resolved via consensus after discussion between two of the authors.

The search revealed 89 manuscripts after removal of duplicates and inappropriate articles. Fifty-five manuscripts were related to prostate gland and coronaviruses. Small cohorts, case reports, comments on guidelines, guidelines, editorials were retrieved. After exclusion, 52 manuscripts were included in the review based on relevance and new data.

The PRISMA flow chart demonstrating the process for the systematic search of the literature and selection of the studies is shown in Fig. 1. Furthermore, Table 2 showed

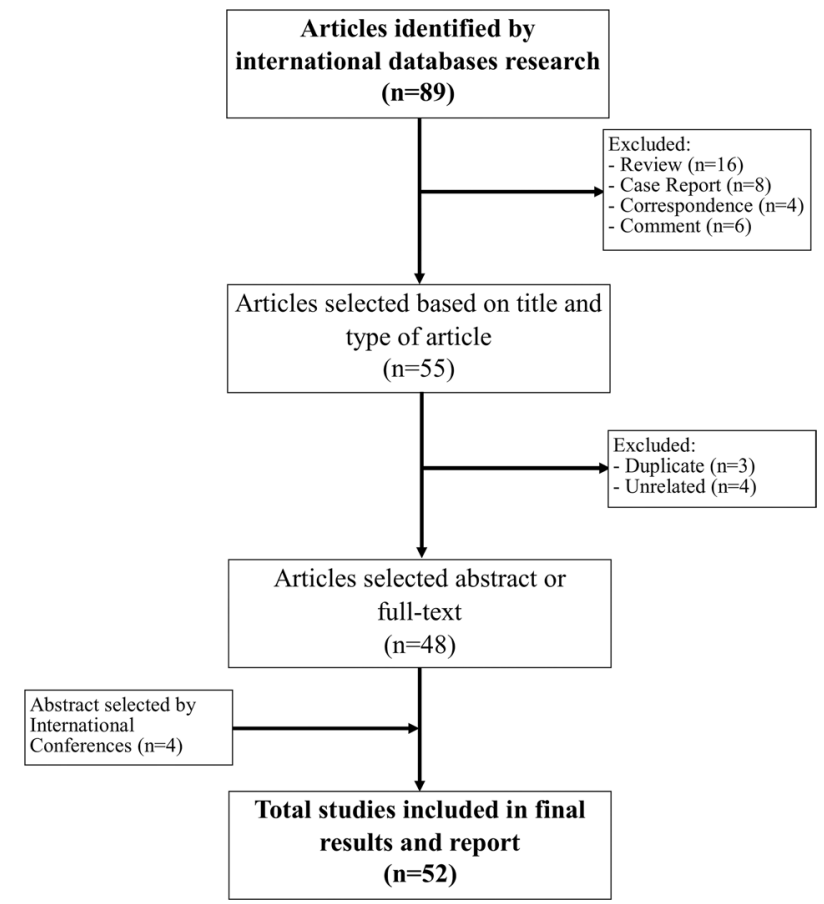

Fig. 1 Article's selection process: the PRISMA flow chart. Literature search according to the Preferred Reporting Items for Systematic Reviews and Meta Analyses (PRISMA) guidelines. grouping of the relevant studies about mechanisms of progression of $\mathrm{BPH}$ as a consequence of SARS-CoV-2 infection.

\section{Results}

\section{Potential mechanism of SARS-CoV-2 infection in the BPH progression through RAS dysregulation}

The renin-angiotensin system (RAS) is a hormonal cascade that regulates the blood pressure and cardiovascular function through its components including angiotensin-II (AngII), and angiotensin converting enzyme (ACE) and RAS hyperactivity is associated with hypertension [22, 23]. Augmented Ang-II level is associated with cellular growth and its activity is mainly mediated by angiotensin-II type 1 receptor (AT1R) [24].

It is well-established that RAS components are locally present in the prostate gland [24, 25]. Ang-II has been detected in epithelial basal layer of the prostate and AT1R was found in the smooth muscle cells of both vessels and the stoma of the prostate gland in some studies [25, 26]. There is evidence that the expression of ACE and Ang-II was markedly increased in patients with BPH. Moreover, AT1R expression is downregulated in patients with BPH due to increased level of Ang-II [26]. Thus, this highlights the potential role of RAS in the development of BPH, and RAS blockade can be suggested as a therapeutic option in patients with $\mathrm{BPH}$.

ACE2 is an enzyme that is known to counterbalance the effects of ACE by cleaving Ang-II to Ang (1-7), a product that binds to Mas receptor. Studies have demonstrated that ACE2 has anti-fibrotic and anti-inflammatory activities and acts as a vasodilator [27-30]. As mentioned before, Ang-II is remarkably increased in $\mathrm{BPH}$ and it has been revealed that Ang-II leads to downregulation of ACE2 and subsequently, a decrease in Ang (1-7) will be observed [26]. Given that Ang-II leads to cellular growth and ACE2-Ang(1-7)/Mas receptor pathway counterbalances ACE and Ang-II functions, the potential role of ACE2 as a novel therapeutic option for treating $\mathrm{BPH}$ [31] is emphasized (Fig. 2).

Studies on SARS-CoV-2 characteristics have delineated that the virus binds to ACE2 receptor, which is expressed in many human tissues such as lung, kidney, prostate, and pancreas, and infects the target tissue. Thus, there is mounting concern about the possibility of the mentioned organs involvement as targets of SARS-CoV-2 [6]. It has been demonstrated that serine protease TMPRSS2 is necessary for priming the viral spike protein and the co-expression of TMPRSS2 and ACE2 is crucial for cell entry. Moreover, it has been suggested that when 


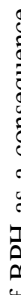

垔

흠

离

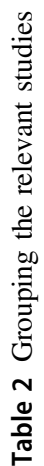

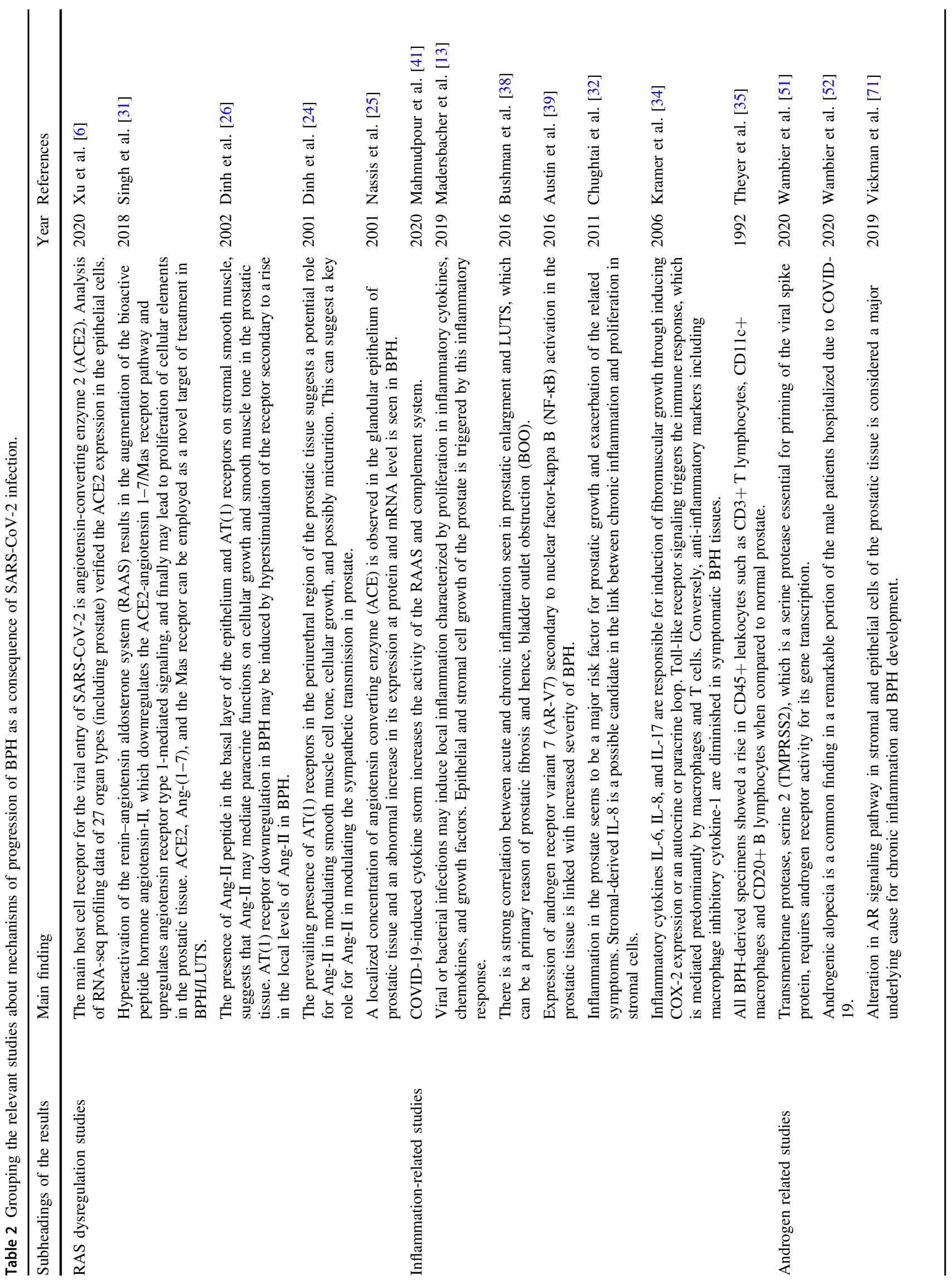

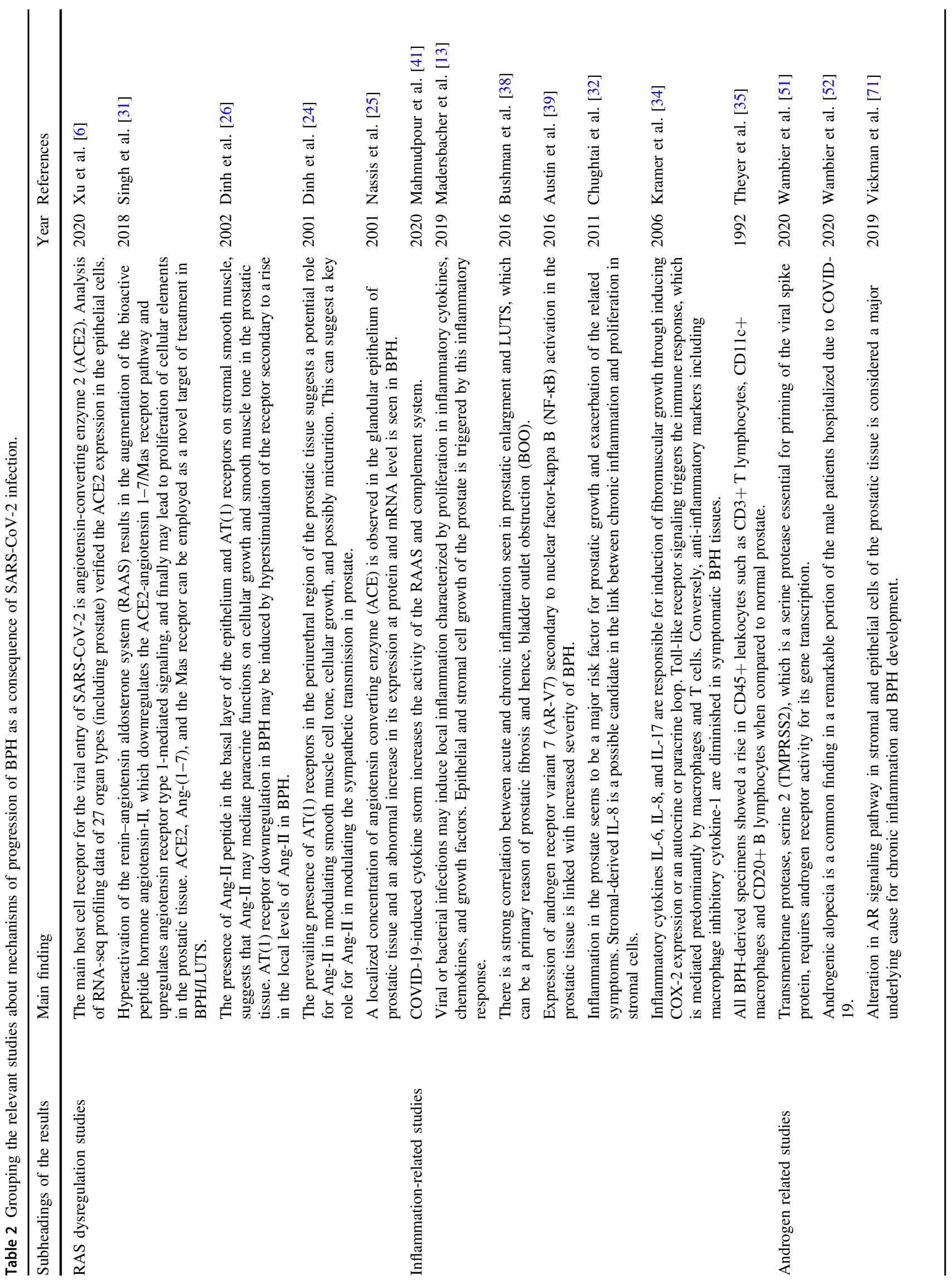

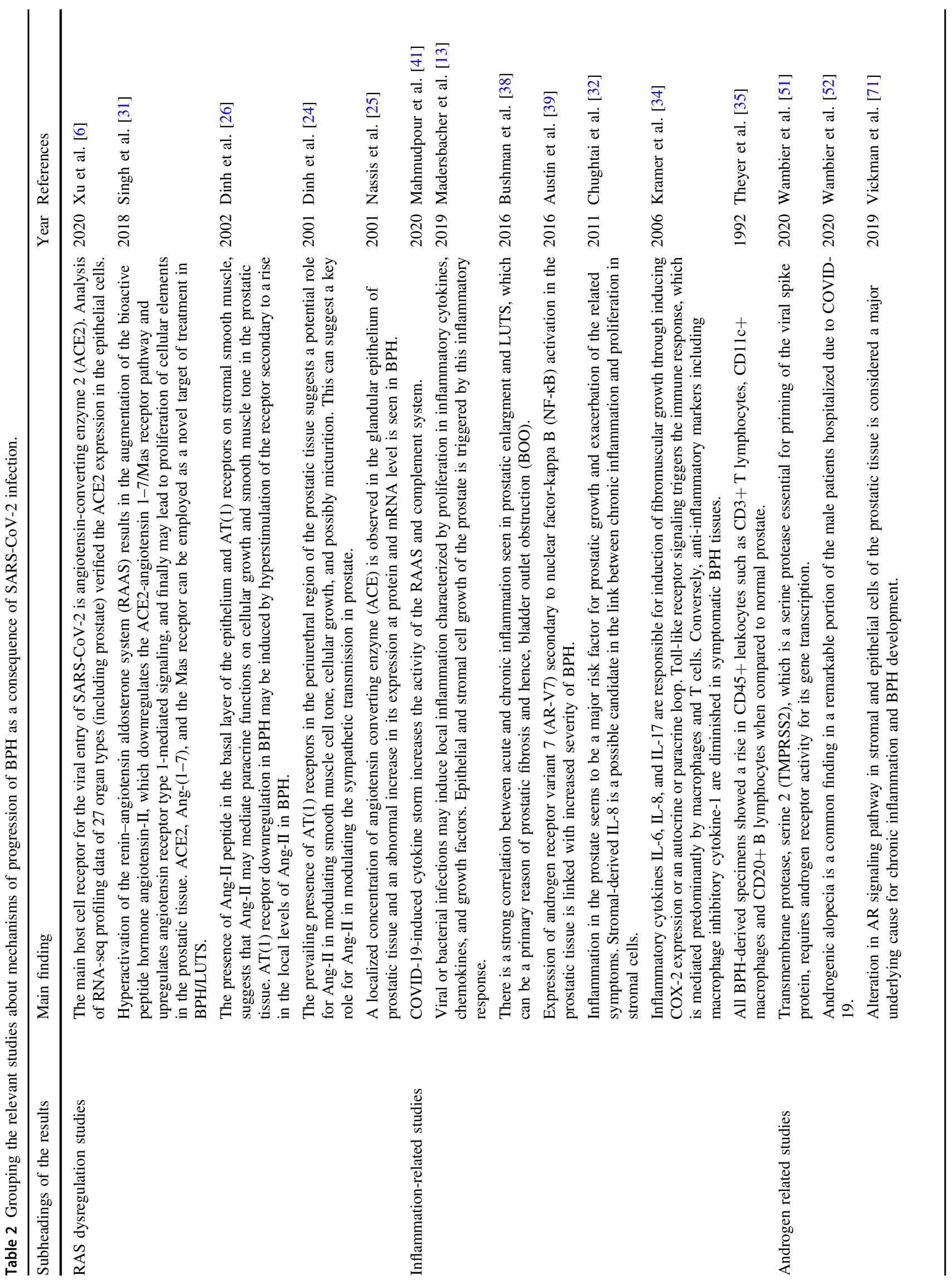




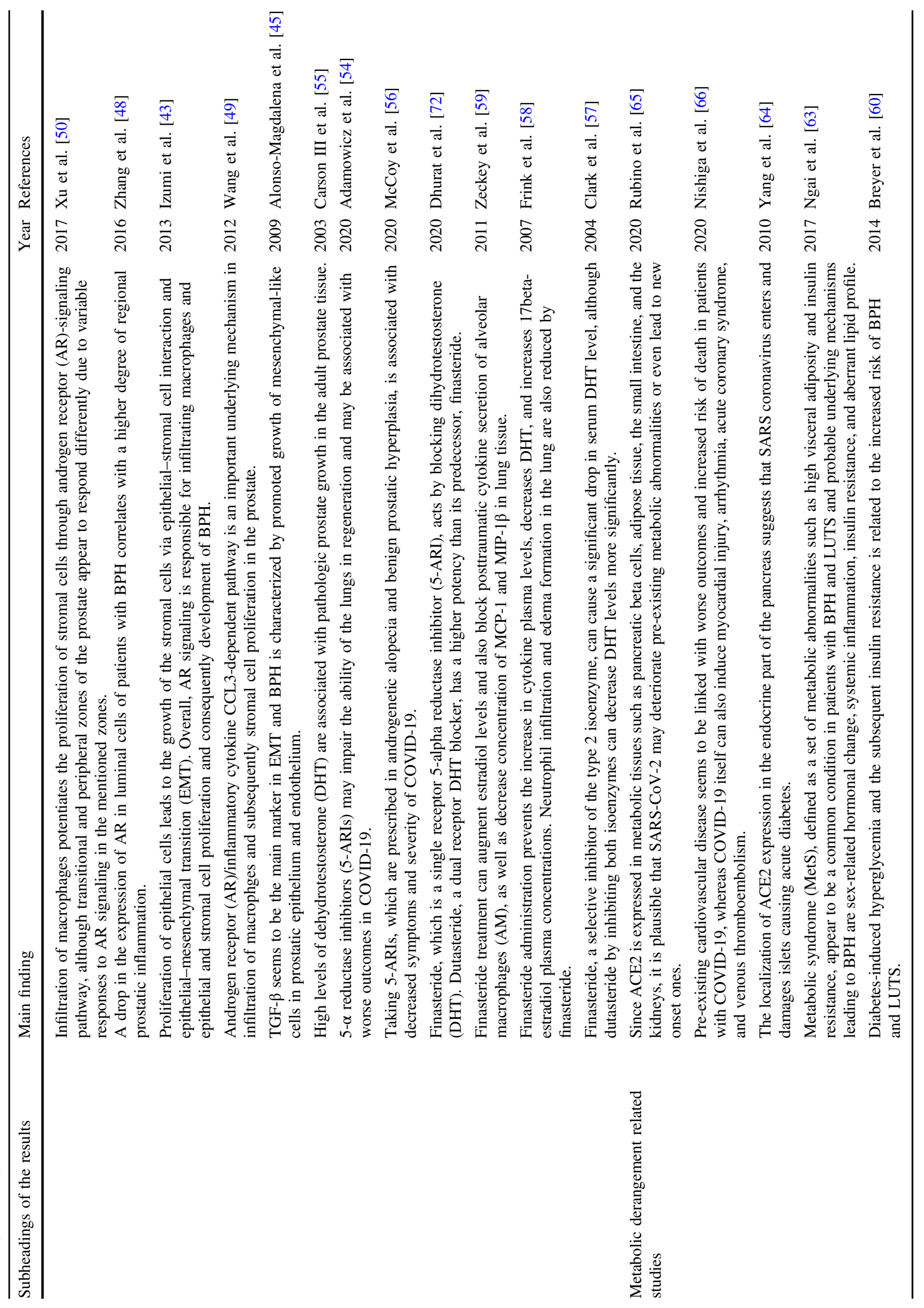




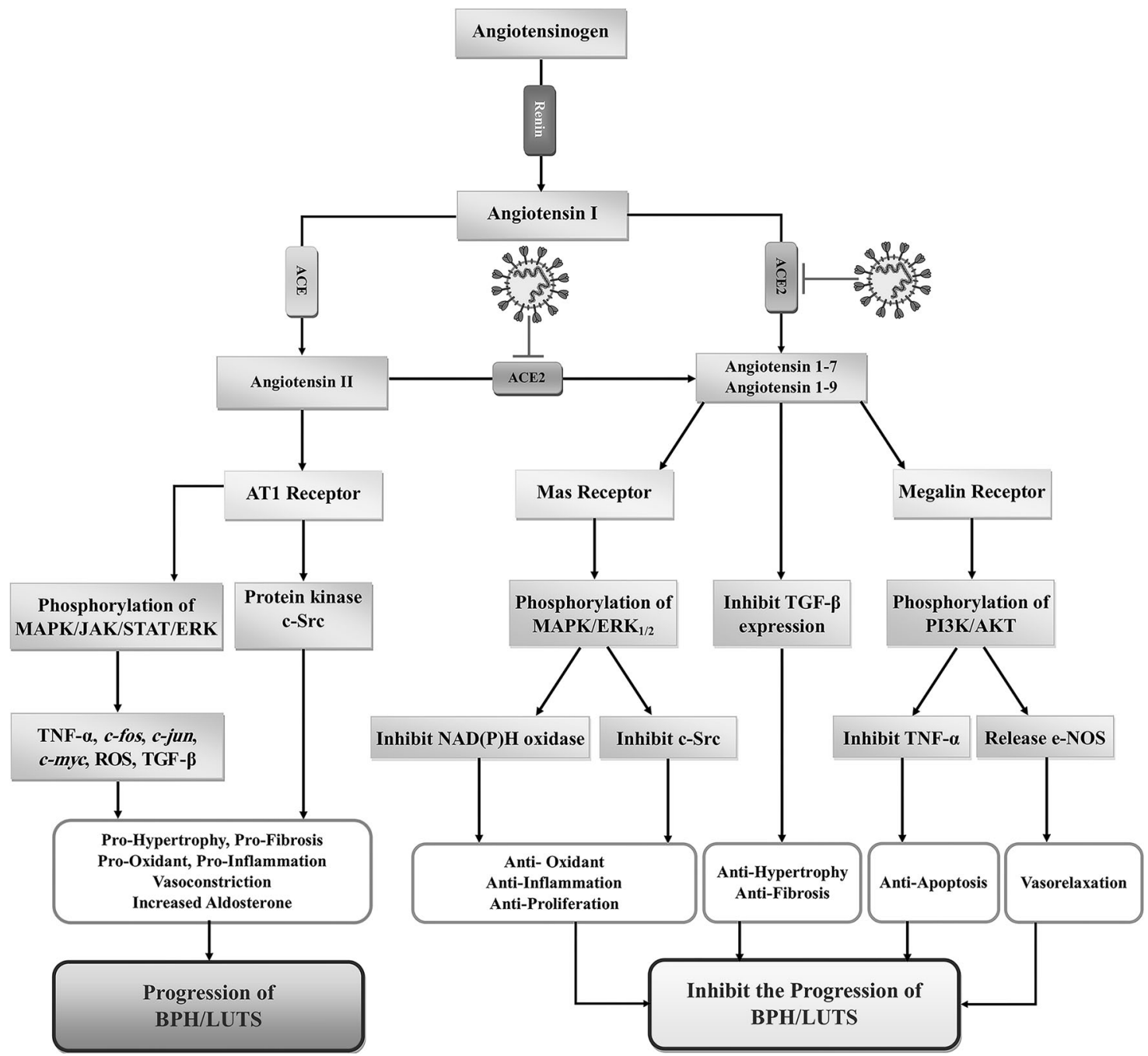

Fig. 2 The role of the ACE2 and the detrimental effect of SARSCoV-2 in the RAAS during progression of BPH/LUTS. Renin cleaves angiotensinogen into angiotensin I (Ang I), and the circulating Ang I is hydrolyzed to Ang-II by ACE. Ang-II activates the AT1R to lead to pro-hypertrophy, pro-fibrosis, pro-oxidant, pro-inflammation, vasoconstrictor response, and to increase aldosterone synthesis. ACE2 directly hydrolyzes Ang I and Ang-II to generate Ang 1-9 and Ang $1-7$, respectively. Ang 1-7 binds to the Mas R/megalin R, which leads

the virus binds to ACE2 receptor through its spike protein, it leads to downregulation of ACE2 [4]. Given the fact that ACE2, which is a component of ACE2/Ang-(1-7)/ Mas system, counteracts the proliferative and inflammatory effects of Ang-II by controlling and inhibiting infection and inflammation it can be concluded that infection with SARS-CoV-2 and a subsequent downregulation of ACE2 can lead to aggravation of a previously diagnosed BPE. Therefore, diminished ACE2 expression and following ACE2 loss of function in the cellular membranes of the prostate tissue, due to viral invasion, can be trigger signals for progression of $\mathrm{BPH}$ (Fig. 2). Consequently, this highlights the urgent need for to the cellular signaling that opposes the Ang-II effects on BPH progression and does not stimulate aldosterone secretion. The SARSCoV-2 disturbs the balance of RAAS by downregulating the ACE2 expression levels. Conclusively, the disproportion between AT1R and Mas $\mathrm{R} /$ megalin $\mathrm{R}$ axes in infected patients with SARS-CoV-2 contributes to the development of BPH/LUTS and more severe inflammatory reactions.

future studies to investigate the possibility of BPH progression as a result of COVID-19.

\section{The possible role of inflammation in the progression of BPH symptoms secondary to SARS-CoV-2 infection}

A correlation between the development of BPH and chronic inflammation has been assumed. This inflammation can be triggered by etiologies such as bacterial or viral infection $[13,32]$. First, bacterial or viral infection can lead to localized prostatic inflammation and subsequently production of different inflammatory cytokines such as IL-6, IL-8, IL10, 


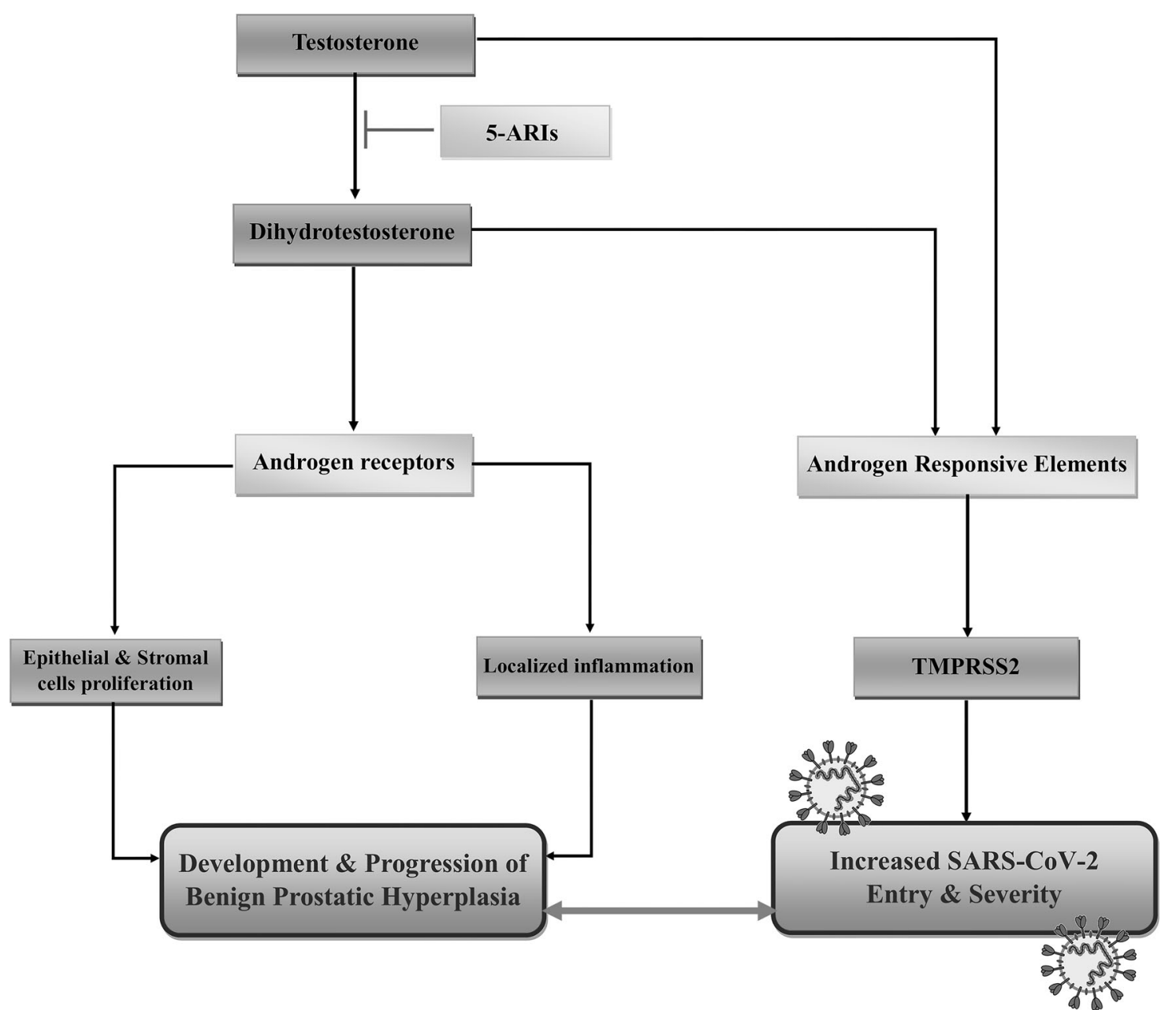

Fig. 3 The story of androgens and androgen receptors (AR) in the development of BPH and severity of COVID-19. There are bidirectional interactions between BPH and COVID-19. Increased production of dihydrotestosterone (DHT) and AR activity through promoting growth in the prostate epithelial and stromal cells and triggering a localized inflammation led to the development and progression of BPH. TMPRSS2, which is an essential serine protease for priming of the spike protein of SARS-CoV-2, requires AR activity and

and TNF- $\beta$ by stromal prostatic cells and infiltrated lymphocytes and macrophages [13, 33-35]. Moreover, it has been demonstrated that the inflammatory process in the prostate can result in the release of self-antigens, trigger an autoimmune response, and subsequently cause tissue damage [36].

It has been suggested that prostatic inflammation is a risk factor for BPH progression [13]. A study has demonstrated that stromal nodules containing a remarkable number of Band T-cell lymphocytes were detected in specimens from patients with $\mathrm{BPH}$, whereas these nodules were not detected in normal prostates [37]. Theyer et al. investigated the characterization of the leukocytes in BPH and found a significant increase in $\mathrm{CD} 45^{+}$leukocytes including macrophages, B-cell, and T-cell lymphocytes were mainly present androgen-responsive elements for its gene transcription. 5-alpha reductase inhibitors (5-ARIs) increase androgen levels and possibly the severity of COVID-19 via inhibiting the conversion of testosterone to dihydrotestosterone. On the other hand, these inhibitors probably reduce the complications of COVID-19 by reducing DHT production, increasing estradiol levels, and suppressing some inflammatory mechanisms.

in the interstitium [35]. Furthermore, inflammatory mediators seem to play a major role in the progression and severity of BPH and LUTS [38]. There is evidence that nuclear factor-kappa $\mathrm{B}(\mathrm{NF}-\mathrm{\kappa B})$ pathway is activated in $\mathrm{BPH}$ patients and is closely associated with the disease severity [39]. Notably, systemic inflammation may contribute to LUTS-related irritative symptoms especially in overweight males [40]. As a result, accumulating evidence suggests the potential role of inflammatory mediators and infiltrated inflammatory cells in BPH progression and severity.

Studies have revealed that ACE2 and its product Ang (1-7) seem to function as an antiproliferative and antiinflammatory agent and modulate leukocyte infiltration and cytokine secretion by counterbalancing the Ang-II effects 
[41]. Moreover, Ang (1-7) seems to have anti-inflammatory effects through suppressing the NF- $\mathrm{KB}$ pathway and cytokines such as IL-6, tumor necrosis factor (TNF)- $\alpha$, and IL-8 $[41,42]$. SARS-CoV-2 induces the suppression of ACE2 in order to gain entry to the target cells [4]. These results provide evidence that infection with SARS-CoV-2 and subsequently suppression of ACE2 may lead to activation of pro-inflammatory pathways, increased cytokine release, and consequently cause inflammatory responses in vulnerable organs such as the prostate. Although the inflammatory mechanism in progression of BPH and its symptoms is still obscure and needs to be elucidated, it can be hypothesized that SARS-CoV-2 can lead to development of irritative symptoms of BPH and trigger inflammatory process in the prostate gland, so prospective studies can help to clarify this issue.

\section{Evidence for the role of androgen receptors in BPH development as a consequence of SARS-CoV-2 infection}

The theory that androgen receptors (AR) play a key role in the development of BPH is well-established [43]. AR are widely expressed in both epithelial and stromal cells of the prostate. First, studies have revealed that AR result in promoting growth in the prostate epithelial cells [44]. Although it has been demonstrated that BPH consists of more stromal cells rather than epithelial cells, proliferation of epithelial cells lead to the growth of the stromal cells via epithelial-stromal cell interaction and epithelial-mesenchymal transition [45]. While there is no definite solidarity regarding any alteration in AR expression throughout $\mathrm{BPH}$ progression, a rise in stromal to epithelial AR ratio is noted in BPH that plays a key role in the progression of BPH [46]. Next, accumulating evidence suggests that AR induce stromal cell proliferation and consequently lead to development of BPH. AR play a role in increased recruitment of migrating macrophages into the prostate stromal cells; therefore, they cause promotion of stromal cell proliferation and subsequently lead to $\mathrm{BPH}$ progression [43]. Contemporary data has assumed that modified AR expression can also contribute to BPH progression by triggering a localized inflammation [47]. A drop in AR expression in prostatic luminal cells followed by a rise in epithelial cells results in local inflammation mostly involving IL-1-dependent pathway. Moreover, stromal cells proliferation in transition zone is related to this localized inflammation via recruitment of macrophages in $\mathrm{C}-\mathrm{C}$ Motif Chemokine Ligand 3-dependent mechanism [48-50] (Fig. 3).

A recent study by Wambier et al. has hypothesized that SARS-CoV-2 infection may be androgen-mediated. It has been suggested that TMPRSS2, which is a serine protease essential for priming of the viral spike protein, requires $A R$ activity for its gene transcription [51] (Fig. 3). Male patients with androgenic alopecia appear to be at greater risk of developing severe cases of COVID-19 and hence, hospitalization supports the hypothesis that COVID-19 is androgen-mediated [52, 53].

As mentioned before, AR are widely expressed in both epithelial and stromal cells of the prostatic tissue and play a major role in the development of BPH [43, 44]. Moreover, infection with SARS-CoV-2 seems to be androgenmediated [51]. Thus, given that AR play a pivotal role in pathophysiology of $\mathrm{BPH}$, one can conclude that causing progression of BPH may be a complication of COVID-19 and further studies should be conducted to investigate this possibility (Fig. 3).

It has been shown that high levels of DHT play a major role in pathophysiology of $\mathrm{BPH}$; thus, decreasing the level of DHT through inhibition of 5-alpha reductase enzyme with agents such as finasteride and dutasteride is a key therapeutic option proposed for BPH treatment [54, 55].

Adamowicz et al. has suggested that 5-alpha reductase inhibitors (5-ARIs) impair the ability of the lungs in regeneration through augmenting the androgen levels in the epithelium of the lungs [54]; thus, it has been hypothesized that patients taking 5-ARIs such as finasteride and dutasteride have more propensity to develop COVID-19 and they may develop more severe cases.

On the other hand, a new study has also revealed that using 5-ARIs may be associated with less severe symptoms of COVID-19, although it may take weeks to reach an effective level in order to decrease DHT [56, 57]. However, caution should be exercised when attempting to draw conclusions about the mechanism of action and efficacy of these drugs that have variable effects on multiple tissues.

The results of previous studies revealed that pretreatment with finasteride reduced the levels of systemic cytokines and pro-inflammatory mediators such as macrophage inflammatory protein- $1 \mathrm{~b}$ and TNF- $\alpha$ released from isolated alveolar macrophages, and increased estradiol levels $[58,59]$. These results suggest that inhibition of 5-alpha reductase leads to the conversion of testosterone to $17 \mathrm{beta}-$ estradiol, which produces salutary effects on the immune response.

Therefore, it can be concluded that the use of these inhibitors on the one hand can result in the deterioration of the COVID-19 progression by increasing androgens, and on the other hand, contribute to the better functioning of the immune system by reducing DHT, increasing estradiol production, and regulating immune responses (Fig. 3).

Overall, controversy exists regarding the role of androgen pathways in the pathophysiology of COVID-19 and this 


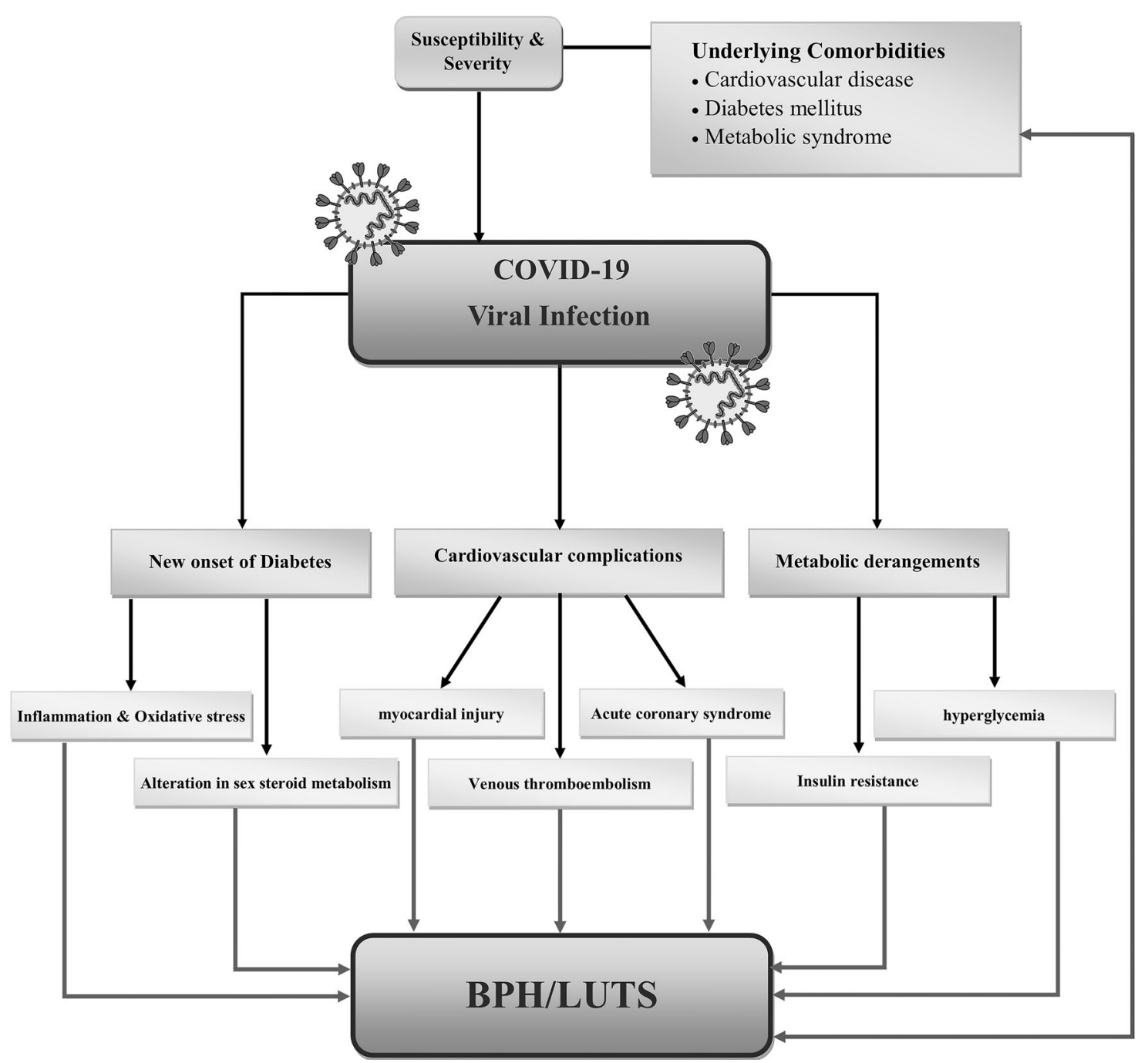

Fig. 4 Bidirectional interaction between BPH and COVID-19. Benign prostatic hyperplasia (BPH) was considered as a possible comorbid condition for COVID-19, due to high prevalence of underlying comorbidities (cardiovascular disease, diabetes mellitus, and metabolic syndrome) in older men with BPH. New onset of diabetes, cardiovascular complications, and metabolic derangement may lead to $\mathrm{BPH}$ development as consequences of SARS-CoV-2 infection. should encourage future studies to clarify the possible effect of anti-androgens on COVID-19 patients.

\section{Evidence for the possible role of diabetes, cardiovascular dysregulation, and metabolic syndrome (MetS) in BPH aggravation and SARS-CoV- 2 infection as a potential culprit}

The possible association between some metabolic disorders including diabetes, cardiovascular diseases, and MetS and development of LUTS has been extensively studied. There is evidence of a propensity for development of BPH and LUTS in patients with diabetes. This can be explained by some factors associated with diabetes-induced hyperglycemia such as proliferation in prostate gland induced by insulin and related trophic factors, sex steroid hormonal change, provoking inflammation and oxidative stress [60]. Furthermore, males diagnosed with hypertension appear to be at a greater risk of more severe LUTS compared to the ones without hypertension [61]. Besides, MetS, which is defined as a set of metabolic abnormalities such as high visceral adiposity and insulin resistance, is a common condition in patients with BPH and LUTS and the majority of the studies support the hypothesis that there seems to be an association between MetS and BPH and its related LUTS [62]. Although the exact underlying mechanism is not fully determined, probable culprits are sex-related hormonal change, systemic inflammation, insulin resistance, and aberrant lipid profile [63].

ACE2, which is the main entry receptor for SARS-CoV2 , is found throughout the metabolic organs such as adipose tissue and pancreatic beta cells, raising concerns regarding 
the possibility of key metabolic organs involvement by the virus and resulting in new onset or exacerbation of preexisting metabolic conditions such as diabetes. Interestingly, there are case reports of newly detected diabetes in patients infected with SARS-CoV [64]. Thus, these findings suggest that COVID-19 may deteriorate pre-existing metabolic abnormalities or even lead to new onset ones including diabetes [65].

Studies have found that patients with an underlying cardiovascular disease are more susceptible to worse outcomes of COVID-19 if infected. Moreover, studies have shown that COVID-19 can cause venous thromboembolism, acute coronary syndrome, and myocardial injury [66].

Taken together, the hypothesis that SARS-CoV-2 can result in new onset comorbid conditions such as diabetes and cardiovascular diseases or exacerbate the previously diagnosed comorbidities has gained recognition. Since these comorbidities are considered a risk factor for developing LUTS and BPH and their pathophysiology are connected in some ways, these findings suggest a potential route for development of LUTS and BPH secondary to exacerbation or new onset development of comorbid metabolic conditions and further studies should investigate this possibility (Fig. 4).

\section{Conclusions and future prospective}

The pandemic caused by the new coronavirus represents an extraordinary scenario in modern medicine that affects many aspects of daily healthcare. Since BPH has a high prevalence and is more common in older men who are more prone to COVID-19, we suggest a closer monitoring of older patients who are more susceptible to both BPH-related LUTS and also COVID-19 infection during this pandemic.

In general, BPH and its related LUTS progress slowly over a long period of time. It is demonstrated that the chance of presenting with acute urinary retention (AUR) in a male diagnosed with moderate-to-severe LUTS is around $0.6-1.8 \%$ per year, with a similar risk of developing infections and bladder stones $[67,68]$. Therefore, this offers a rationale for monitoring the progression of symptoms of patients with BPH and LUTS during the COVID-19 pandemic.

The observation of patients with BPE is largely consisted of several aspects. We recommend the use of available questionnaires such as IPSS to identify the degree of bother [69]. As elevated serum PSA level is one of the most proven predictors of AUR episodes in patients with BPH, checking the serum PSA during and after pandemic in patients with old age affected by COVID-19 seems to be reasonable [70].

There are no studies investigating the potential of BPH progression as a complication of SARS-CoV-2 infection at the present time and only a few studies have proposed BPH management during the SARS-CoV-2 pandemic. However, our literature review showed that different mechanisms such as ACE2 signaling alteration, AR-related mechanisms, inflammation, and metabolic derangement might lead to aggravation of BPH-related LUTS and its complications (e.g., AUR) over and after the course of infection with SARS-CoV-2.

Overall, since infection with SARS-CoV-2 seems to be an unraveled challenge for the human being, a deeper investigation regarding the possibility of COVID-19 leading to exacerbation of BPH symptoms and worsening of the previously diagnosed condition may help the scientists and clinicians discover novel mechanisms of infection of this disease in addition to better management of it.

Acknowledgements The authors wish to thank Dr Nasrin Shokrpour, professor of English at Shiraz University of Medical Sciences, and Ms Sheryl Thomas-Nikpoor, Language Editor, Springer Publications for her invaluable comments in editing this paper. We also appreciate Center for Development of Clinical Research of Namazi Hospital for their helpful assistance.

Author contributions A Ha, FM, and A Ho researched data for the article; MS and JR made substantial contributions to discussions of content; A Ha, FM, A Ho, and AD wrote the article; and MS and JR reviewed and edited the manuscript before submission.

Funding This study was supported by the Vice Chancellor of Research Affairs, Shiraz University of Medical Sciences (Academic Grant Number: 99-01-18-23730).

\section{Compliance with ethical standards}

Conflict of interest The authors declare no competing interests.

Publisher's note Springer Nature remains neutral with regard to jurisdictional claims in published maps and institutional affiliations.

\section{References}

1. World Health Organization. Coronavirus disease 2019 (COVID19): situation report. 88. 2020.

2. Wang $\mathrm{D}, \mathrm{Hu} \mathrm{B}, \mathrm{Hu} \mathrm{C}$, Zhu F, Liu X, Zhang J, et al. Clinical characteristics of 138 hospitalized patients with 2019 novel coronavirus-infected pneumonia in Wuhan, China. JAMA. 2020;323:1061-9.

3. Gu J, Han B, Wang J. COVID-19: gastrointestinal manifestations and potential fecal-oral transmission. Gastroenterology. 2020;158:1518-9.

4. Chai X, Hu L, Zhang Y, Han W, Lu Z, Ke A, et al. Specific ACE2 expression in cholangiocytes may cause liver damage after 2019nCoV infection. bioRxiv. 2020. https://doi.org/10.1101/2020.02. 03.931766.

5. Hoffmann M, Kleine-Weber H, Schroeder S, Krüger N, Herrler T, Erichsen S, et al. SARS-CoV-2 cell entry depends on ACE2 and TMPRSS2 and is blocked by a clinically proven protease inhibitor. Cell. 2020;181:271-80.e8. 
6. Xu H, Zhong L, Deng J, Peng J, Dan H, Zeng X, et al. High expression of ACE2 receptor of 2019-nCoV on the epithelial cells of oral mucosa. Int J Oral Sci. 2020;12:1-5.

7. Foo KT. Pathophysiology of clinical benign prostatic hyperplasia. Asian J Urol. 2017;4:152-7.

8. Lepor H. Pathophysiology of benign prostatic hyperplasia in the aging male population. Rev Urol. 2005;7:S3-S12.

9. Abrams P. LUTS, BPH, BPE, BPO: a plea for the logical use of correct terms. Rev Urol. 1999;1:65.

10. Capogrosso P, Salonia A, Montorsi F. Evaluation and nonsurgical management of benign prostatic hyperplasia. In: Campbell-WalshWein urology. 12th ed. Philadelphia, PA: Elsevier; 2021.

11. Roehrborn CG. Male lower urinary tract symptoms (LUTS) and benign prostatic hyperplasia (BPH). Med Clin. 2011;95: 87-100.

12. Porru D, Jallous H, Cavalli V, Sallusto F, Rovereto B. Prognostic value of a combination of IPSS, flow rate and residual urine volume compared to pressure-flow studies in the preoperative evaluation of symptomatic BPH. Eur Urol. 2002;41:246-9.

13. Madersbacher S, Sampson N, Culig Z. Pathophysiology of benign prostatic hyperplasia and benign prostatic enlargement: a minireview. Gerontology. 2019;65:458-64.

14. Berry SJ, Coffey DS, Walsh PC, Ewing LL. The development of human benign prostatic hyperplasia with age. J Urol. 1984;132:474-9.

15. Li LQ, Huang T, Wang YQ, Wang ZP, Liang Y, Huang TB, et al. COVID-19 patients' clinical characteristics, discharge rate, and fatality rate of meta-analysis. J Med Virol. 2020;92:577-83.

16. Liang X. Is COVID-19 more severe in older men? Postgrad Med J. 2020;96:426.

17. Mizumoto K, Kagaya K, Zarebski A, Chowell G. Estimating the asymptomatic proportion of coronavirus disease 2019 (COVID19) cases on board the Diamond Princess cruise ship, Yokohama, Japan, 2020. Eur Surveill. 2020;25:2000180.

18. Nishiura H, Kobayashi T, Miyama T, Suzuki A, Jung S-m, Hayashi $\mathrm{K}$, et al. Estimation of the asymptomatic ratio of novel coronavirus infections (COVID-19). Int J Infect Dis. 2020;94:154-5.

19. Can O, Erkoç M, Ozer M, Umeyir Karakanli M, Otunctemur A. The effect of COVID-19 on lower urinary tract symptoms in elderly men. Int J Clin Pract. 2021;75:e14110.

20. Kaya Y, Kaya C, Kartal T, Tahta T, Tokgöz VY. Could LUTS be early symptoms of COVID-19. Int J Clin Pract. 2020;75:e13850.

21. Moher D, Liberati A, Tetzlaff J, Altman DG. Preferred reporting items for systematic reviews and meta-analyses: the PRISMA statement. PLoS Med. 2009;6:e1000097.

22. Cohn JN. Role of the renin-angiotensin system in cardiovascular disease. Cardiovasc Drugs Ther. 2010;24:341-4.

23. Peach MJ. Renin-angiotensin system: biochemistry and mechanisms of action. Physiol Rev. 1977;57:313-70.

24. Dinh DT, Frauman AG, Sourial M, Casley DJ, Johnston CI, Fabiani ME. Identification, distribution, and expression of angiotensin II receptors in the normal human prostate and benign prostatic hyperplasia. Endocrinology. 2001;142:1349-56.

25. Nassis L, Frauman AG, Ohishi M, Zhuo J, Casley DJ, Johnston CI, et al. Localization of angiotensin-converting enzyme in the human prostate: pathological expression in benign prostatic hyperplasia. J Pathol. 2001;195:571-9.

26. Dinh DT, Frauman AG, Somers GR, Ohishi M, Zhou J, Casley DJ, et al. Evidence for activation of the renin-angiotensin system in the human prostate: increased angiotensin II and reduced AT1 receptor expression in benign prostatic hyperplasia. J Pathol. 2002;196:213-9.

27. Guy JL, Jackson RM, Acharya KR, Sturrock ED, Hooper NM, Turner AJ. Angiotensin-converting enzyme-2 (ACE2): comparative modeling of the active site, specificity requirements, and chloride dependence. Biochemistry. 2003;42:13185-92.
28. Mizuiri S, Ohashi Y. ACE and ACE2 in kidney disease. World J Nephrol. 2015;4:74-82.

29. Santos RA, Ferreira AJ, Simões e Silva AC. Recent advances in the angiotensin-converting enzyme 2-angiotensin (1-7)-Mas axis. Exp Physiol. 2008;93:519-27.

30. Silveira KD, Barroso LC, Vieira AT, Cisalpino D, Lima CX, Bader M, et al. Beneficial effects of the activation of the angiotensin-(1-7) MAS receptor in a murine model of adriamycininduced nephropathy. PLoS ONE. 2013;8:e66082.

31. Singh Y, Gupta G, Sharma R, Matta Y, Mishra A, Pinto TdJA, et al. Embarking effect of ACE2-angiotensin 1-7/mas receptor axis in benign prostate hyperplasia. Crit Rev Eukaryot Gene Expr. 2018;28:115-24.

32. Chughtai B, Lee R, Te A, Kaplan S. Role of inflammation in benign prostatic hyperplasia. Rev Urol. 2011;13:147-50.

33. Dinarello CA. Historical insights into cytokines. Eur J Immunol. 2007;37:S34-45.

34. Kramer G, Marberger M. Could inflammation be a key component in the progression of benign prostatic hyperplasia? Curr Opin Urol. 2006;16:25-9.

35. Theyer G, Kramer G, Assmann I, Sherwood E, Preinfalk W, Marberger $\mathrm{M}$, et al. Phenotypic characterization of infiltrating leukocytes in benign prostatic hyperplasia. Lab Invest. 1992;66:96.

36. Soler R, Andersson K-E, Chancellor MB, Chapple CR, de Groat WC, Drake MJ, et al. Future direction in pharmacotherapy for non-neurogenic male lower urinary tract symptoms. Eur Urol. 2013;64:610-21.

37. Bierhoff E, Vogel J, Benz M, Giefer T, Wernert N, Pfeifer U. Stromal nodules in benign prostatic hyperplasia. Eur Urol. 1996;29:345-54.

38. Bushman WA, Jerde TJ. The role of prostate inflammation and fibrosis in lower urinary tract symptoms. Am J Physiol-Ren Physiol. 2016;311:F817-21.

39. Austin DC, Strand DW, Love HL, Franco OE, Jang A, Grabowska $\mathrm{MM}$, et al. NF- $\mathrm{KB}$ and androgen receptor variant expression correlate with human BPH progression. Prostate. 2016;76:491-511.

40. Freedland SJ, Aronson WJ. Invited commentary: lower urinary tract symptoms and inflammation-weighing the evidence. Am J Epidemiol. 2009;169:1291-5.

41. Mahmudpour M, Roozbeh J, Keshavarz M, Farrokhi S, Nabipour I. COVID-19 cytokine storm: the anger of inflammation. Cytokine. 2020;133:155151.

42. Yu X, Cui L, Hou F, Liu X, Wang Y, Wen Y, et al. Angiotensinconverting enzyme 2-angiotensin (1-7)-Mas axis prevents pancreatic acinar cell inflammatory response via inhibition of the p38 mitogen-activated protein kinase/nuclear factor- $\kappa \mathrm{B}$ pathway. Int $\mathrm{J}$ Mol Med. 2018;41:409-20.

43. Izumi K, Mizokami A, Lin W-J, Lai K-P, Chang C. Androgen receptor roles in the development of benign prostate hyperplasia. Am J Pathol. 2013;182:1942-9.

44. Kyprianou N, Davies P. Association states of androgen receptors in nuclei of human benign hypertrophic prostate. Prostate. 1986;8:363-80.

45. Alonso-Magdalena P, Brössner C, Reiner A, Cheng G, Sugiyama $\mathrm{N}$, Warner $\mathrm{M}$, et al. A role for epithelial-mesenchymal transition in the etiology of benign prostatic hyperplasia. Proc Natl Acad Sci. 2009; 106:2859-63.

46. Bauman DR, Steckelbroeck S, Peehl DM, Penning TM. Transcript profiling of the androgen signal in normal prostate, benign prostatic hyperplasia, and prostate cancer. Endocrinology. 2006;147:5806-16.

47. Aaron-Brooks LM, Sasaki T, Vickman RE, Wei L, Franco OE, Ji $\mathrm{Y}$, et al. Hyperglycemia and $\mathrm{T}$ cell infiltration are associated with stromal and epithelial prostatic hyperplasia in the nonobese diabetic mouse. Prostate. 2019;79:980-93. 
48. Zhang B, Kwon OJ, Henry G, Malewska A, Wei X, Zhang L, et al. Non-cell-autonomous regulation of prostate epithelial homeostasis by androgen receptor. Mol Cell. 2016;63:976-89.

49. Wang X, Lin WJ, Izumi K, Jiang Q, Lai KP, Xu D, et al. Increased infiltrated macrophages in benign prostatic hyperplasia (BPH): role of stromal androgen receptor in macrophage-induced prostate stromal cell proliferation. J Biol Chem. 2012;287:18376-85.

50. Xu D, Wang X, Jiang C, Ruan Y, Xia S, Wang X. The androgen receptor plays different roles in macrophage-induced proliferation in prostate stromal cells between transitional and peripheral zones of benign prostatic hypertrophy. Excli J. 2017;16:939-48.

51. Wambier CG, Goren A. Severe acute respiratory syndrome coronavirus 2 (SARS-CoV-2) infection is likely to be androgen mediated. J Am Acad Dermatol. 2020;83:308-9.

52. Wambier CG, Vaño-Galván S, McCoy J, Gomez-Zubiaur A, Herrera S, Hermosa-Gelbard Á, et al. Androgenetic alopecia present in the majority of patients hospitalized with COVID-19: the "Gabrin sign". J Am Acad Dermatol. 2020;83:680-2.

53. Lamy PJ, Rébillard X, Vacherot F, de la Taille A. Androgenic hormones and the excess male mortality observed in COVID-19 patients: new convergent data. World J Urol. 2020;1-3.

54. Adamowicz J, Juszczak K, Drewa T. May patients receiving 5alpha-reductase inhibitors be in higher risk of COVID-19 complications? Med Hypotheses. 2020;140:109751.

55. Carson C, Rittmaster R. The role of dihydrotestosterone in benign prostatic hyperplasia. Urology. 2003;61:2-7.

56. McCoy J, Cadegiani FA, Wambier CG, Herrera S, Vaño-Galván S, Mesinkovska NA, et al. 5-alpha-reductase inhibitors are associated with reduced frequency of COVID-19 symptoms in males with androgenetic alopecia. J Eur Acad Dermatol Venereol. 2020;35:e243-6.

57. Clark RV, Hermann DJ, Cunningham GR, Wilson TH, Morrill BB, Hobbs S. Marked suppression of dihydrotestosterone in men with benign prostatic hyperplasia by dutasteride, a dual 5alpha-reductase inhibitor. J Clin Endocrinol Metab. 2004;89:2179-84.

58. Frink M, Hsieh YC, Hu S, Hsieh CH, Pape HC, Choudhry MA, et al. Mechanism of salutary effects of finasteride on posttraumatic immune/inflammatory response: upregulation of estradiol synthesis. Ann Surg. 2007;246:836-43.

59. Zeckey C, Andruszkow H, Neunaber C, Frink M, Schirmer B, Mommsen $\mathrm{P}$, et al. Protective effects of finasteride on the pulmonary immune response in a combined model of traumahemorrhage and polymicrobial sepsis in mice. Cytokine. 2011;56:305-11.
60. Breyer BN, Sarma AV. Hyperglycemia and insulin resistance and the risk of BPH/LUTS: an update of recent literature. Curr Urol Rep. 2014;15:462.

61. Sugaya K, Kadekawa K, Ikehara A, Nakayama T, Gakiya M, Nashiro F, et al. Influence of hypertension on lower urinary tract symptoms in benign prostatic hyperplasia. Int $\mathrm{J}$ Urol. 2003;10:569-75.

62. De Nunzio C, Aronson W, Freedland SJ, Giovannucci E, Parsons JK. The correlation between metabolic syndrome and prostatic diseases. Eur Urol. 2012;61:560-70.

63. Ngai HY, Yuen KS, Ng CM, Cheng CH, Chu SP. Metabolic syndrome and benign prostatic hyperplasia: an update. Asian $\mathrm{J}$ Urol. 2017;4:164-73.

64. Yang JK, Lin SS, Ji XJ, Guo LM. Binding of SARS coronavirus to its receptor damages islets and causes acute diabetes. Acta Diabetol. 2010;47:193-9.

65. Rubino F, Amiel SA, Zimmet P, Alberti G, Bornstein S, Eckel $\mathrm{RH}$, et al. New-onset diabetes in Covid-19. N Engl J Med. 2020;383:789-90.

66. Nishiga M, Wang DW, Han Y, Lewis DB, Wu JC. COVID-19 and cardiovascular disease: from basic mechanisms to clinical perspectives. Nat Rev Cardiol. 2020;17:543-58.

67. McConnell JD, Roehrborn CG, Bautista OM, Andriole GL Jr., Dixon CM, Kusek JW, et al. The long-term effect of doxazosin, finasteride, and combination therapy on the clinical progression of benign prostatic hyperplasia. N Engl J Med. 2003;349:2387-98.

68. Roehrborn CG, Bruskewitz R, Nickel GC, Glickman S, Cox C, Anderson R, et al. Urinary retention in patients with BPH treated with finasteride or placebo over 4 years. Characterization of patients and ultimate outcomes. The PLESS Study Group. Eur Urol. 2000;37:528-36.

69. Barry MJ, Fowler FJ Jr., O’Leary MP, Bruskewitz RC, Holtgrewe HL, Mebust WK, et al. The American Urological Association Symptom Index for benign prostatic hyperplasia. $\mathrm{J}$ Urol. 2017;197:S189-97.

70. Roehrborn CG. Acute urinary retention: risks and management. Rev Urol. 2005;7:S31-41.

71. Vickman RE, Franco OE, Moline DC, Vander Griend DJ, Thumbikat P, Hayward SW. The role of the androgen receptor in prostate development and benign prostatic hyperplasia: a review. Asian J Urol. 2020;7:191-202.

72. Dhurat R, Sharma A, Rudnicka L, Kroumpouzos G, Kassir M, Galadari $\mathrm{H}$, et al. 5-Alpha reductase inhibitors in androgenetic alopecia: shifting paradigms, current concepts, comparative efficacy, and safety. Dermatol Ther. 2020;33:e13379. 